\title{
XIV.
}

\section{Thiersch'sche Transplantation nach der Aufmeisselung des Warzenfortsatzes.}

(Vortrag, gehalten in der otologischen Section der 70. Naturforscherversammlung in Düsseldorf a. Rh. September 1898.)

Von

Dr. Karl Reinhard in Duisburg.

Wenn man die heutige Literatur in unseren otologischen Zeitsehriften über die Nachbehandlung des aufgemeisselten Warzenfortsatzes verfolgt, so fällt einem ins Auge, das ein grosser Theil der Publicationen die Lappenbildung behandelt, durch welche die gesehaffenen Wundfächen möglichst schnell überhäutet werden sollen. Und auch das Programm der diesjährigen Versammlung zeigt, dass die Vorschläge über Plastik und Lappenbildung nach der Radicaloperation noch lange nicht erschöpft sind.

Wo so viel Angebot des Guten ist, hat man in unserer medicinischen Wissenschaft stets zu befürchten, dass, weil eben so viele Vorschläge gemacht sind, noch keiner bisher den idealen Anforderungen genügte. Es giebt wohl keine Epidermisstelle um und in der Nähe der Mittelohrräume, welche nicht schon von Meisterhand als Deekfläche für den neu geschaffenen Wundkanal benutzt wäre. Namen wie Sehwartze, Kretsehmann, Stacke, Panse, Körner, u. a. m. müssen hier genannt werden. Alle haben versucht, aus den vorhandenen an die Wundhöhle anstossenden natürlichen Epidermisgrenzen Lappen in der verschiedensten Art zu bilden und diese durch Verlagerung, Naht und Tamponade zur Bedeckung der einzelnen Wundfächen zu benutzen, um dadurch eine sehnellere Epidermisirung der geschaffenen grossen Wunde herbeizufuhren. Am idealsten erschien noch die Idee von Panse-Körn er, welche einen Lappen aus der hinteren Gehörgangswand bildeten und diesen erstens 
durch primäre Naht der retroauriculären Oeffnung und zweitens durch Tamponade sofort nach der Operation auf die hintere Wand des Knochenkanales anwachsen liessen, wodurch vier Epidermisränder geschaffen waren, von denen aus die erforderte Epidermisirung stattfinden konnte. Die Nachbehandlung fand dann durch den dilatirten Meatus auditorius externus statt. Leider eignet sich diese Methode nicht für alle Fälle. Denn wenn auch Jansen auf der diesjährigen Versammlung der deutsehen otologischen Gesellsehaft in Würzburg mittheilte, dass er diese Methode selbst für anfangs nicht recht geeignet ersoheinende Fälle anwende, d. h. für solehe, bei denen grössere Complicationen der chronischen Mittelohreiterung bestanden, wie z. B. epitympanischer, perisinuöser Abscess, Defect im horizontalen Bogengange, so bin ich doch der Ansicht, dass es zum mindesten nicht sehr praktisch erscheint, in solchen Fällen, wo dem Operateur die Beschaffenheit der weiteren intracraniellen Organe, besonders des Sinus noch unbekannt ist, durch sofortigen Verschluss der eben geschaffenen Wundhöhle die directe Controle des Auges über die fernere Beschaffenheit der Wundhöhle sich zu entziehen.

Wenn es auch mit Hülfe des Spiegels und des Trichters möglich ist, bei einer regelrecht ausgefübrten Radicaloperation alle Punkte der Wundhöhle vom Meatus aus zu beobachten, so sind bei diesem Verfahren eventuell nothwendig werdende spätere chirurgische Eingriffe ganz besonders erschwert, resp. nur nach Wiederöffnen des primären Verschlusses möglich. Ich halte es in keinem Falle für absolut sicher möglich, nach Beendigung der Radicaloperation mit Bestimmtheit sagen zu können: alles Kranke vom Knochen ist entfernt. In jedem Falle können kleine cariöse Stellen, entfernt gelegene kranke Zellen, z. B. oberhalb des Labyrinths, der Labyrinthwand selbst, der hinteren Antrumswand, am Boden der Paukenhöhle ete, bei der Operation ubersehen sein. Und gerade diese sind es, welehe der Nachbehandlung so grosse Schwierigkeit entgegensetzen. Hat man dann nach dem Panse-Körner'schen Verfahren einen primären retroauriculären Verschluss geschaffen, so ist die Freude selbst über die prima intentio gering. Deshalb halte ich es fuir nothwendig, in der Wahl dieser Methode vorlänfig noch vorsichtig zu sein. Ich wende sie selbst in einer grossen Zahl meiner Radicaloperationen an und bekenne offen, dass, wenn die Auswahl eine richtige war, $d . h$. wenn es sich um superficielle 
Caries im Atticus oder im Antrum oder um Caries der Gehörknöchelchen handelte, der Eirfolg ein eclatanter war, and die Nachbehandlung bedentend abgekürzt wurde. Die Patienten werden früher von ihrem Verbande befreit, treten früher in ambulatorische Behandlung ein und werden früher ihrer Berufsthätigkeit wiedergegeben. Selbstverständlich ist es, dass der Erfolg gerade in diesen Fällen von strengster Asepsis abhängig ist.

Was soll nun geschehen mit den Fällen - und diese bilden doch vorläufig noch die Mehrzahl -, in welchen man sich zu einer primären Naht direct im Anschluss an die Radicaloperation nicht hat entschliessen können. Zuerst waren Siebenmann und fast zu gleicher Zeit auch ich diejenigen, welche auf das allgemein bekannte Verfahren der Transplantation von Epidermis nach Thiersch auf granulirende Flächen bei dem eröffneten Warzenfortsatz aufmerksam gemacht haben. Si ebenmann (Berliner Klinische Wochenschrift $1893 \mathrm{Nr}$. 1) verpflanzte Thiersch'sche Läppchen nach Ablauf von 3-4 Wochen nach der Radicaloperation in den aufgemeisselten Warzenfortsatz hinein, zugleich mit Bildung von Lappen aus der Kopfhaut, in der Absicht, auf diese Weise eine persistente Oeffnung zu erhalten. Tch habe im Jahre 1893 bereits versucht, Thiersch'sche Epidermis-Läppchen direet im Anschluss an die Aufmeisselung auf die geschaffenen Wundfächen zu übertragen, bin aber nach unerfreulichen Resultaten in kurzer Zeit wieder hiervon abgekommen. Den Grund dieser schlechten Resultate fuhre ich, abgesehen von noch mangelhafter Technik, darauf zurück, dass niemals nach der Operation mit Sicherheit constatirt werden konnte, $o b$ alles Kranke des Knochens entfernt war; meistens waren es Fälle von ausgedehnter Zerstörung. der Wände, die bei der Operation nicht vollkommen beseitigt war, bei denen der Misserfolg eintrat.

Maassgebend waren für mich zwei Gesichtspunkte: erstens soll durch die Einführung von Epidermisstückehen von ferner gelegenen Körperstellen eine schnellere Ueberhäutung der Wundhöhle erzielt werden, und zweitens soll vor allem der Aditus ad antrum und das Antrum selbst gegen den äusseren Gehörgang hin weit und bequemer, als es durch die bisher itbliche Tamponade zu erreichen war, offen gehalten werden. Bekanntlich ist es gerade die letztere Stelle, welche durch rasche Granulationswucherung trotz eifriger Tamponade so leicht wieder ver- 
wächst und sich dem Alige somit entzieht. Ist die Radicaloperation beendet und die Plastik aus der häutigen hinteren Gehörgangswand nach Stacke, resp. Panse ausgeführt, so tamponire ich in der bekannten Weise die ganze Wundhöhle, wechsle je nach der Temperaturcurve und der Stärke der Secretion den Verband am 3., 4. oder auch 5. Tage und entschliesse mich, entweder sofort beim ersten Verbandweehsel, oder beim zweiten oder auch dritten, bei denen die Granulationen schon üppig emporschiessen und keine Neigung zum Bluten zeigen, zur Transplantation nach Thierseh. Die dann vorhandenen Granulationen werden zunächst in Narkose sanft entfernt, und die Wundböhle wird hierauf von neuem tamponirt. Die Blutung steht hierbei rasch. Während die Tampons liegen, werden aus einer Stelle des Oberarmes oder des Beines genau nach der Vorschrift von Thierseh genügende Epidermisstreifen von der Länge von $2-4 \mathrm{~cm}$ und ungefähr $1 \mathrm{~cm}$ Breite excidirt und in blutwarmer physiologischer Kochsalzlösung aufgehoben. Es werden so viele Hautstückchen genommen, als zur Bedeckung aller granulirenden Flächen nöthig erscheint. Auf die Schrumpfung: der Läppchen nach'ihrer Excision wird dabei von vornherein Rücksicht genommen. Je dünner der Lappen, desto schmiegsamer ist er, desto besser "klebt" er fest. Die neugeschaffene Hautwunde an der betreffenden Extremität wird sogleich mit trockner steriler Gaze derart verbunden, dass der Verband ungefähr drei Wochen liegen bleiben kann, nach welcher Zeit stets eine vollständige Ueberhäutung stattgefunden hat. Ueber intensive Sohmerzen an den Stellen, denen die Epidermis entnommen war, wurde, ausser direct nach der Operation, niemals geklagt, so dass der Verband stets bis zur vollständigen Heilung: liegen bleiben konnte. Mein Assistent breitet nun die einzelnen Epidermisstreifen auf besondere Spatel ${ }^{1}$ ) aus, von denen ich Ihnen hier einige zeige, und die sich von den Jansen'schen Spateln durch eine grössere Breite an ihrer Basis zur Aufnahme grösserer Läppohen unterscheiden. Inzwischen sind die Tampons aus der Wundhöhle vorsichtig entfernt, mit grösstmöglichster Vermeidung wieder eintretender Blutung. Die Lappen werden nun mit Hülfe der Spatel derart in den Wundtrichter eingelegt, dass der Spatel zunächst auf die hintere Antrumswand gebracht und das auf dem Spatel liegende Läppehen durch Fortziehen des

1) Zu haben bei Instrumentenmacher Johnen-Duisburg, Beeckstr. 13 . 
Spatels auf diese Stelle glatt aufgelegt wird. Die folgenden Läppchen werden in gleicher Weise an das eben eingelegte Läppchen derart eingeführt, dass stets eins das andere dachziegelartig deckt. Auf diese Weise versucht man, die ganze Wundhöhle, auch die mediale und obere Paukenwand, sowie den vorderen Wundrand mit Epidermishäutchen zu bedecken. Falls sich an der medialen Paukenhöhlenwand cariöse Stellen vorgefunden haben, wird der Thiersch'sche Lappen von hinten her aus dem Antrum so weit mit der Sonde nach vorn gesehoben, dass er auch diese Wundflächen, die bei der Operation natürlich mit dem scharfen Löffel behandelt sind, bedeckt. Die nach aussen liegenden Läppehen ragen bis auf die Epidermis der Kopfhaut heraus, so dass es den Anschein hat, als ob man eine persistente retroauriculäre Oeffnung schaffen wolle. Letzteres ist aber nicht die Absicht in jedem Falle, sondern geschieht, wie tiberhaupt die ganze Bedeckung der granulirenden Flächen aus dem Grunde, die Secretion der Granulationen, welche in den nächsten Tagen entsteht und immer mehr zunimmt und die Läppchen sehr leicht wieder abspülen würde, möglichst zu vermeiden, resp. zu verringern. Will man keine retroauriculäre Oeffnung erzielen, so ist später durch Wiederanfrischen der Wundränder leicht ein Verschluss wiederherzustellen.

Nachdem nun auf diese Weise die Wundfläche vollständig mit Thiersch'schen Läppchen bedeckt ist, werden konisch zugeschnittene aseptische, feuchte Silkprotectivstücke von der Länge und Breite meiner Spatel auf die Thierseh'schen Läppchen in den Wundtrichter wiederum so eingelegt, dass sie sich dachziegelartig uberragen. Die Wunde wird nun nicht tamponirt, sondern es werden abweichend von der bisherigen Methode einige kleine Verbandgazestreifen sowohl durch den Gehörgang, als auch durch die retroauriculäre Oeffnung in die Wundhöhle eingelegt, um das Wundsecret anzusangen. Dann kommen darauf zur guten Unterlage für die Ohrmuschel sowohl nach vorn wie nach hinten einige feuchte Gazetampons, und das Ganze wird nochmals mit Protectivs bedeckt und der Verband so angelegt, dass sich die Ohrmuschel möglichst nicht bewegen kann.

Die Silkprotectivstreifen müssen auch bei den Verbänden innerhalb der ersten 8-14 Tage jedesmal wieder auf die Läppehen gelegt werden. Dadurch wird das Anhaften einzelner 
Läppchen am Verbande beim Wechsel desselben mit Sicherheit vermieden. Der erste Verbandwechsel wird am zweiten oder dritten Tage, je nach derStärke der Secretion oder bei Temperaturerhöhung früher vorgenommen, wobei man darauf zu achten hat, dass noeh nicht angeheilte Thiersch'sche Läppchen in ihrer Lage verbleiben, da selbst nach Verlauf von mehreren Tagen die Anheilung noch erfolgen kann. Anfangs sehen die Läppehen ödematös durchtränkt und gelblich verfärbt aus und erwecken den Eindruck, als ob alle Mühe vergebens gewesen wäre. Dies ist aber keineswegs der Fall. Sobald man beim Verbandweehsel sieht, dass ein Läppchen oder ein Stück eines Läppchens nekrotisch zu werden beginnt and sich von der Unterfläche loslöst, muss dasselbe durch vorsichtiges Spülen oder mit der Scheere entfernt werden. Doch hüte man sich, festsitzende Theile mit zu entfernen.

Was nun den Erfolg dieser Methode anbelangt, so ist derselbe im wesentlichen von 2 Punkten abhängig, und zwar erstens von der richtigen Auswahl der Fälle und zweitens von der manuellen Fertigkeit des Operateurs. Je vorsichtiger man in der Auswahl der Fälle ist, und je sicherer man die Technicismen, welche oft sebr viel Geduld erfordern, beherrseht, desto schöner ist der Erfolg. Dort, wo kranker Knochen bei der Operation stehen geblieben war, oder wo, wie es bei Tuberculose sehr häufig vorkommt, durch die sogenannte punktförmige Caries (Infiltrationstuberculose $[\mathrm{K} \ddot{0} \mathrm{nig}]$ ) in einem bislang gesunden Gebiete plötzlich kranke Stellen auftreten, wird der Epidermislappen entweder überhaupt nicht anheilen, oder er wird kurz oder lang unterminirt, und eine neue Fistel wird sich bilden. Man soll sich selbst durch viele Misserfolge nioht abhalten lassen, stets wieder von neuem Epidermis nach Thierseh in die Wundhöhle einzupflanzen. Die vielen Mühen, welche das Verfahren selbst mit sich bringt - das oftere Sichverschieben der Lappen in der Wundhöhle, die Schwierigkeit des Fernhaltens von Flüssigkeit in der Tiefe während des Hineinbringens der Läppchen - werden reichlich wieder ansgeglichen durch die $\mathbf{A b}$ kürzung der Behandlung, welche für die Folge auch bedeutend weniger Belästigung für den Patienten mit sich bringt. Denn wenn es auch äusserst schwierig ist zu erreichen, dass alle Läppchen anheilen, so gelingt es doch fast stets, an einzelnen Stellen Epidermisinseln zu schaffen, von denen aus eine schnellere Ueberhäutung der Wundhöhle stattfindet, als durch das Tampo- 
192 XIV. REINHARD, Thiersch'sche Transpl. nach d. Aufmeissel. u. s. w.

nadeverfahren. Und selbst in den Fällen, wo die Läppchen nicht anheilen, sondern sich nach 8-14 Tagen als nekrotische Fetzen wieder abstossen, beobachtet man, dass sich unter den abgestossenen Häutchen kleine weissgraue Epithelpunkte bilden, von welchen unter Verminderung der Secretion rascher als bislang die Wundhöhle zur Ueberhäutung kommt. Deshalb möchte ich empfehlen, dass das Thi e r s c h'sche Verfahren bei der Aufmeisselung des Warzenfortsatzes sich trotz der oft recht sehwierigen Technicismen immer mehr Freunde erwerbe, damit so die so segensreiche Radicaloperation nicht durch die Schrecken einer langen Nachbehandlung eingeschränkt werde. 